\title{
Laparoscopic Removal of Retained Artery Forceps Causing Internal Hernia
}

\author{
Saleh Alzahrani ${ }^{*}$, Awadh Alqahtani \\ King Abdulaziz Medical City National Guard Hospital -Riyadh \\ King Khalid Hospital - King Saud University -Riyadh
}

Received: 04 October, 2016; Accepted: 20 October, 2016; Published: 29 October, 2016

*Corresponding author: Saleh Alzahrani, Department of Surgery, king Abdulaziz Medical City National Guard Hospital -Riyadh, Tel no: 96604378613; E-mail: smz0022@hotmail.com

\begin{abstract}
Retained foreign bodies are a surgical complication resulting from foreign materials accidently left in a patient's body. It is rare but potentially dangerous error. It is also under-reported and carries serious medico-legal consequences.

Here, we present an interesting case of retained artery forceps post laprotomy and total abdominal hysterectomy bilateral salpengioophrectomy and ventral hernia repair treated by laparoscopy.

Conclusion: Retained instruments in intra-abdominal surgery can cause serious complication. It should be treated surgically. Laparoscopic exploration and removal can be done safely. Preventive measures against retained instruments must follow strict protocols for surgical instruments handling in theatre.

Keywords: Retained intra-abdominal artery forceps; Intestinal obstruction; Intestinal strangulation; Laparoscopy; Case report
\end{abstract}

\section{Introduction}

Retained foreign body in the abdominal cavity following surgery is a recurrent problem. However the incidence is grossly underestimated [2] and rarely reported [3]. The rarity of reported cases may be due to medico - legal implications or the surgeon's unwillingness to advertise his errors. The incidence of retained foreign body in literature is 1 per 1000 to 1500 laparotomies [4]. Retained foreign body in peritoneal cavity is one of the known but avoidable complications of abdominal surgery. It is more liable to occur when surgery is done in a non-ideal environment and negligence or ignorance on part of the medical personnel. It is the responsibility of the operating surgeon and the theatre staff to ensure that no foreign body is left inside the peritoneal cavity before closure of the abdominal cavity. Retained foreign bodies are of various types and include sponges/gauzes, artery forceps, other metal instruments, surgical needles, surgical drains, and rubber tubes. The surgical sponges/gauzes are the most common retained foreign body because of its common usage, small size and amorphous structure [5] Retained artery forceps are much rarer. Retained artery forceps remain inert and induce their effects through mechanical means giving rise to pressure effects resulting in abdominal pain or direct tissue/viscous injury leading to tissue strangulation or perforation of viscous leading to for example peritonitis or vascular injury resulting in hemorrhage [1]. The clinical presentation of retained intra-abdominal foreign material may be acute or delayed $[6,7]$. Such patient present in the surgical units with increasing abdominal pain, abdominal mass, discharging sinus, intra-abdominal abscesses and acute or subacute intestinal obstruction. Whilst many patients will present within days or few weeks after the initial surgery and have a relaprotomy for retrieval of the foreign body, some patients with intra-abdominal foreign material may go unnoticed for years or even decades. However many of this subset of patients will have chronic abdominal symptoms such as abdominal pain or discomfort. The pre-operative diagnosis is based on high index of suspicion. The pre-operative diagnosis is based on history, clinical examination, plain abdominal radiographs, ultrasound, CT and MRI scans [1]. Management of retained material should be surgical through exploration and save removal. Laparoscopic exploration and removal was not published before for artery forceps. We present a case of retained artery forceps during laprotomy for uterine mass and hernia repair which was managed successful by laparoscopy.

\section{Case Report}

56 year old housewife women presented to emergency department with complain of three days of abdominal pain, vomiting and distension. She is three weeks status post laprotomy for huge uterine mass and ventral hernia. She underwent total abdominal hysterectomy bilateral salpengioophrectomy and on lay mesh repair of ventral hernia. Post operative course was un eventual. She was discharged day five post operatively. Upon presentation to emergency department she had no fever, no urinary or respiratory symptoms. History of constipation. No other associated symptoms. Examination showed calm patient dehydrated with stable vital sign. Abdominal examination 
showed distended abdomen with midline healed wound and localize peritonitis in right iliac and supra-pubic area. Bowel sound was hyperactive. Rectal exam showed soft stool no blood. Other clinical examination was unremarkable. Laboratory work showed only White blood cell of 15,000 others were normal. Imaging showed retained forceps intra-abdominally [Figure 1-2]. She was diagnosis with retained artery forceps causing small bowel obstruction. Laparoscopic exploration was established through right upper quadrant access using verse needle and total of three $5 \mathrm{~mm}$ trocars. Adehesolysis and exposing artery forceps done by running the small bowel. There was small bowel loop herniated through the opening of jaw of forceps causing obstruction (internal hernia) [figure1]. Opening of the forceps jaws laparoscopiclly released the obstruction. Bowel loops were healthy and there was no resection. Retrieval of forceps through right upper quadrant by extending the wound under vision. Post operative course was uneventful and patient discharged day five.

\section{Discussion}

Retained foreign body in a patient after surgery is an uncommon but dangerous error and it is grossly under estimated [2]. However many of the cases of retained foreign body are not reported [3]. The rarity of the reported cases may be due to medico-legal reasons. In literature the incidence of retained
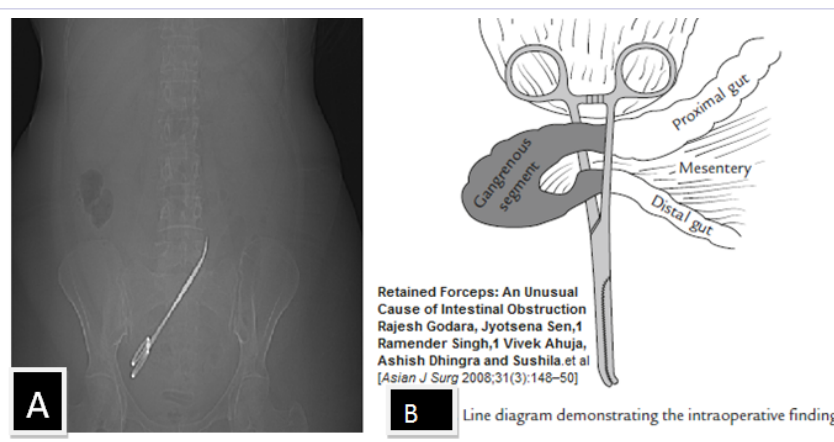

Figure 1: (A) Plan film showed artery forceps. (B) illustrated digram showed mechanism of bowel obstruction caused by artery forceps.

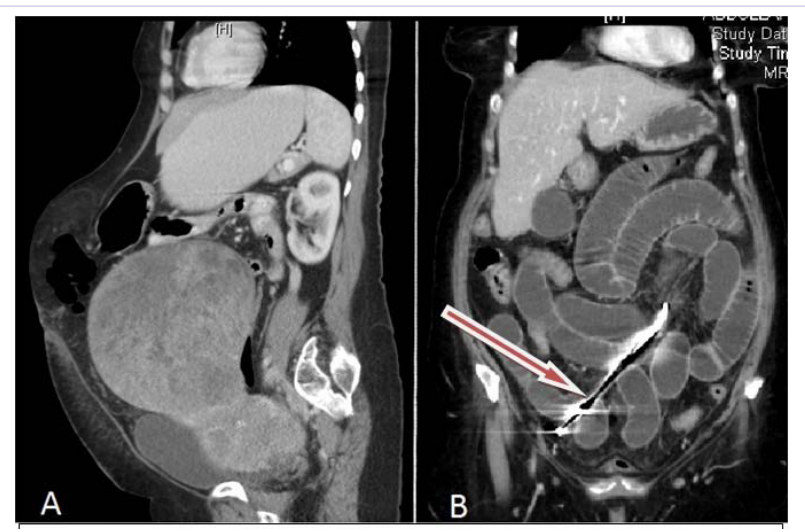

Figure 2: (A) C.T scan sagittal view of preoperative finding (uterine mass and ventral hernia).

(B) Coronal view C.T showed bowel obstruction arrow pointing to artery forceps. foreign body is quoted as 1: per 1000 to 1500 laparotomies [4]. In our environment, the incidence is unknown because no local data exist. The most common retained foreign body is abdominal sponge because of its common usage and amorphous structure [5]. The clinical presentation of retained materials may be acute or delayed [6, 7]. While many retained foreign materials are identified and retrieved immediately or shortly after surgery some may remain unidentified for years or decades [8, 9]. Intraabdominal retained artery forceps are much rare. Retained metal like artery forceps are inert and exert their effect through mechanical means like direct tissue damage causing ischemia, pressure effect giving rise to pain and discomfort [10] or forming an axis on which loops of bowel entangle leading to intestinal obstruction and strangulation or a loop of bowel going through the 'eye' of the retained artery forceps and getting trapped leading to strangulation [11]. They may also penetrate hollow organs for example gut leading to peritonitis or blood vessels leading to hemorrhage. In our case internal hernia of bowel loops through instrument was the cause of obstruction. A retrospective study found that retained instruments and sponges following an operation occurred more frequently in emergency surgery, especially where there was an unplanned change in the operative procedure, in patients with high mean body mass index $[3,13]$. Others noted that huge intra-abdominal tumors or mass are risk factors as in our case. Other risk factors are fatigued surgical team, staff changes during operation, and failure to account for all sponges and instruments, leaving closure of abdominal wound to a junior, sometimes inexperienced [14]. In a case we present, there was two teams operating gynecology and general surgery. Exploratory laprotomy remains the mainstay of treatment to retrieve retained intra-abdominal foreign material. Laparoscopic removal is minimal invasive approach to avoid laprotomy especially post hernia repair with mesh. It allows better exploration and avoid midline repair. Post operative adhesion are limiting factor for completing laparoscopic exploration. But it is not always dense. In our case were manageable especially the hernia repair was on lay. Extracting instrument was challenging as we worked with five mm troacr. Right upper quadrant trocar site was extended and the forceps removed under vision.

\section{Conclusion}

A case of retained large artery forceps following hysterectomy and hernia repair is presented. Retained foreign body is avoidable and could be a costly error.. All preventive protocols must be observed under the strict guidance and full responsibility of the surgeon. The need to investigate properly a patient who complains of chronic abdominal symptoms following laparotomy should be encouraged as this will aid in making early diagnosis of retained foreign material before more serious complications of intestinal obstruction or strangulation, or peritonitis, intraabdominal abscesses or enterocutanous fistula supervenes.

\section{References}

1. Ugochukwu AI, Edeh AJ. Retained intra-abdominal artery forceps - an unusual cause of intestinal strangulation. North Am J Med Sci. 2011;3(7):339-343. doi: 10.4297/najms.2011.3339. 
2. Grag M, Aggarawal AD. A review of medico legal consequences of gossypiboma. J Indian Acad Forensic Med. 2010;32(4):358-361.

3. Gawande AA, Student DM, Orar EJ, Brennan TA, Zinner MJ. Risk factors for retained instruments and sponges after surgery. N Engl J Med. 2003;348(3):229-235.

4. Rappaport W, Hayness K. The retained surgical sponge following intra abdominal surgery: A continuing problem. Arch Surg. 1990;125(3):405-407.

5. Lincourt AE, Harrell A, Cristiano J, Sechrist C, Kercher K, Heniford BT. Retained foreign bodies after surgery. J Surg Res. 2007;138(2):170174.

6. Gonzalez-Ojeda A, Rodriguez-Alcantar DA, Arenas-Marquez H, Sanchez Perez-Verdia E, Chavez-Perez R, Alvarez-Quintero R, et al. Retained foreign bodies following intra abdominal surgery. Hepatogastroenterology. 1999;46(26):808-812.

7. Wang CF, Cipolla J, Seamon MJ, Lindsey DE, Stawicki SP. Gastrointestinal complications related to retained surgical foreign bodies (RSFB): a concise review. OPUS 12 Scientist. 2009;3(1):11-18.

8. Roumen RM, Weerdenburg HP. MRI features of a 24-year old gossypiboma. A case report. Acta Radiol. 1988;39(2):176-178.
9. Hadrami J , Rojas M, de Fenoyl O , Kharsa G, Marzelle J , Chotard Y. Pulmonary textiloma reviewed by haemoptysis 12 years after thoracotomy. Rev Med Intern. 1998;19(11):826-829. doi: 10.1016/ S0248-8663(98)80388-3.

10. Nasir GAA. Missed instruments and surgical sponge (gauze and pack) The Internet Journal of Surgery. 2008;20(1):3-9.

11. Fadiora SO, Olatoke SA, Bello To, Adeoti ML, Agodirin SO. Intestinal obstruction from forgotten artery forceps, a case report. West Afr J Med. 2004;23(4):330-331.

12. Godara R, Sen J, Singh R, Ahuja V, Dhingra A, Godara S. Retained forceps: An unusual cause of intestinal obstruction. Asian J Surg. 2008;31(3):148-150.

13. Wang CF, Stawicki SP, Cook CH, et al. Proceedings of the Central Surgical Association Annual Meeting, Sarasota. Florida (March 7, 2009)

14. Murad M, Bari V. Gossypiboma - a case report. Pak J Med Sci. 2003;19:138-140.

15. Uranus S, Schauer C, Pfeifer J, Dagdoglu A. Laparascopic removal of large laparatomy pad forgotten in site. Surg Laparosc Endosc. 1995;5(1):77-79. 